\title{
Estudo do Amido de Farinhas Comerciais Comestíveis
}

\author{
Bianca N. B. Lima, Thayná B. Cabral, Roberto P. C. Neto, Maria Inês B. Tavares \\ Instituto de Macromoléculas Professora Eloisa Mano, UFRJ \\ Anna Paola T. Pierucci \\ Instituto de Nutrição Josué de Castro, UFRJ
}

Resumo: $\mathrm{O}$ amido em quatro tipos de farinhas comerciais para uso doméstico em preparações culinárias foi caracterizado pelas técnicas de espectroscopia de absorção na região do infravermelho com Transformada de Fourier (FTIR), difração de raios X (DRX), análise termogravimétrica e ressonância magnética nuclear de baixo campo, por meio da determinação do tempo de relaxação spin-rede do núcleo de hidrogênio $(\mathrm{T} 1 \mathrm{H})$.

Palavras-chave: Amido, biopolímeros em alimentos, farinhas comerciais, RMN de baixo campo.

\section{Characterization of Commercial Edible Starch Flours}

Abstract: Four commercial flour samples with starch components were studied. They were characterized by Infrared spectroscopy, X-ray diffraction, thermogravimetric analysis and low field NMR $(\mathrm{T} 1 \mathrm{H})$.

Keywords: Starch, food biopolymers, commercial flour, low field NMR.

\section{Introdução}

O amidoéum polissacarídeo constituído por unidades repetitivas de D-glicopiranose, no qual as unidades de D-glicose estão unidas por ligações $\alpha-1,4$. $\mathrm{O}$ amido é formado por dois componentes: a amilose e a amilopectina, a amilose é essencialmente linear e a amilopectina é uma cadeia ramificada (ligações $\alpha-1,6$ entre a cadeia principal e as ramificações $)^{[1-4]}$. Como polissacarídeo de reserva energética vegetal, o amido está presente na forma de pequenos grânulos nos tecidos fotossintéticos em muitos tipos de órgãos de armazenamento tais como sementes, caules e raízes.

O amido é o polissacarídeo mais importante e abundante encontrado em alimentos. Principal carboidrato da dieta humana, o amido, fornece de vinte a cinquenta por cento do valor total de energia consumido diariamente em uma dieta balanceada ${ }^{[5]}$.

A legislação brasileira, em portaria do Ministério da Saúde, determina que a denominação amido seja referente ao polissacarídeo de reserva de partes aéreas vegetais e fécula ao polissacarídeo proveniente das partes subterrâneas dos vegetais. A farinha é o produto obtido pela moagem da parte comestível de vegetais, podendo sofrer previamente processos tecnológicos adequados ${ }^{[6]}$.

A espectroscopia de RMN fundamenta-se na absorção seletiva de ondas de radiofrequiência por um núcleo colocado em um campo magnético externo e forte. Esta espectroscopia pode ser realizada tanto em espectrômetro de alto campo (300 a $900 \mathrm{MHz})$, como de baixo campo (1 a $23 \mathrm{MHz}$ ), que se diferenciam pela força do campo magnético aplicado, ou seja, quanto maior a força do campo magnético, maior será a sensibilidade (resolução espectral) do equipamento ${ }^{[7-11]}$.

Os equipamentos de baixo campo magnético são muito utilizados para determinação da dinâmica molecular de alimentos, por meio da relaxação nuclear do núcleo de hidrogênio. Devido à sua alta abundância isotópica para esta determinação não há a necessidade de obter espectros, pois são determinados os valores dos parâmetros de relaxação ${ }^{[12-17]}$. Por meio deste parâmetro pode-se estabelecer a dinâmica molecular das amostras de amido e de acordo com valores determinados pode-se inferir a forma da cristalinidade do amido como A, B ou C, esses dados juntamente com os dados de raios $\mathrm{X}$ permitem entender melhor sua solubilidade, cozimento e etc. Os valores de tempo de relaxação spin-rede do núcleo de hidrogênio traduzem o comportamento da dinâmica molecular em alimentos dada às mudanças na organização estrutural, devida à perda ou eliminação de água quando submetido ao processo de cozimento. A eliminação de água promove ou propicia mudanças nas interações intermoleculares com relação às interações de hidrogênio entre as cadeias. $\mathrm{O}$ aumento no tempo de relaxação pode ser função de um aumento na força de interação intermolecular entre as cadeias. As formas cristalinas possuem tempos de relaxação distintos em face das diferentes organizações moleculares distintas ${ }^{[7-17]}$.

O objetivo deste trabalho foi a caracterização e o estudo de farinhas amiláceas comerciais para uso doméstico de quatro diferentes fontes, disponíveis no mercado brasileiro.

\section{Experimental}

As farinhas comestíveis para uso doméstico foram obtidas em supermercados e lojas varejistas localizadas na cidade do Rio de Janeiro-RJ, Brasil. Foram estudadas as farinhas de amido de milho, fécula de batata, banana verde e trigo, por técnica de espectroscopia de absorção na região do infravermelho (FTIR), difração de raios X (DRX), análise termogravimétrica (TG) e tempo de relaxação spin-rede $\left(\mathrm{T}_{1} \mathrm{H}\right)$.

\section{Determinação do teor de umidade e cinzas}

A determinação do teor de umidade e do teor de cinzas foram realizadas segundo as Normas Analíticas do Instituto Adolfo Lutz $(1985)^{[18]}$ : em triplicatas, a partir de $5 \mathrm{~g}$ de amostra colocadas em cápsula de porcelana.

\section{Análises de espectroscopia de absorção na região do infravermelho}

As amostras de farinha foram submetidas à análise em espectrofotômetro de absorção na região do infravermelho Varian, modelo Excalibur 3100 FT-IR no modo de transmitância, com 
acúmulo de 20 varreduras e resolução de $2 \mathrm{~cm}^{-1}$. Cada amostra foi macerada e homogeneizada com $\mathrm{KBr}$ para a formação de pastilhas usadas para a aquisição dos espectros.

\section{Análises de difração de raios $X$}

Os difratogramas de raios $\mathrm{x}$ das farinhas comerciais foram obtidos em um Difratômetro de raios-X - Rigaku - Modelo Miniflex operado a $30 \mathrm{kV}, 15 \mathrm{~mA}$, com um passo de 0,05 e taxa de varredura de $1 \%$ min na temperatura ambiente. A faixa de varredura da difração foi ajustada para ângulos de $5^{\circ}$ a $40^{\circ}(2 \theta)$.

\section{Análises termogravimétricas}

As análises termogravimétricas foram realizadas em analisador termogravimétrico modelo TGA-7 Perkin Elmer na faixa de 25 a $500{ }^{\circ} \mathrm{C}$ com varredura de $10{ }^{\circ} \mathrm{C} / \mathrm{min}$. em atmosfera de $\mathrm{N}_{2}$.

\section{Determinação do grau de cristalinidade}

O porcentual de cristalinidade das amostras foi obtido pelo método de ajuste dos picos dos difratogramas de raios $\mathrm{x}$, através do software estatístico Fityk versão 0.9.3 (GNU General Public License - 2010). O modelo matemático utilizado para o ajuste das gaussianas em cada difratograma foi o de Levenberg-Marquardt. Considerou-se para os cálculos a razão do somatório das áreas dos picos cristalinos pela área total de todas as gaussianas de acordo com a Equação 1. Em que: Xc é o grau de cristalinidade, Ic o somatório das áreas dos picos cristalinos e Ia a área do halo amorfo.

$$
X c(\%)=I c /(I c+I a) * 100
$$

\section{Medidas de relaxação nuclear $\left(T_{1} H\right)$}

Para a determinação das medidas de relaxação nuclear foi utilizado um espectrômetro de RMN de baixo campo magnético Maran Ultra 23, operando à frequência de 23,4 MHz (para núcleos de hidrogênio) e equipado com sonda de $18 \mathrm{~mm}$ à temperatura de $25^{\circ} \mathrm{C}$. O tempo de relaxação spin-rede do hidrogênio foi determinado diretamente pela sequência de pulso inversão-recuperação $\left(180^{\circ}-\tau-90^{\circ}\right)$, com um pulso de $90^{\circ}$ calibrado automaticamente para $4,2 \mu$ s. O intervalo de $\operatorname{tau}(\tau)$ foi de 0,1 a $5000 \mathrm{~ms}$, composto de 40 medições resultantes da média de 4 medidas para cada uma. O tempo de espera entre cada pulso foi de 5 segundos. Os valores do tempo de relaxação e as intensidades de cada domínio foram obtidos pelo fitting dos dados exponenciais através do software WinFit (versão 2.4.0.0).

\section{Resultados e Discussão}

A umidade representa a água contida no alimento, que pode ser classificada em: umidade de superfície, que se refere à água livre ou presente na superfície externa do alimento, facilmente evaporada e umidade adsorvida, referente à água ligada, encontrada no interior do alimento, sem combinar-se quimicamente com o mesmo. A umidade corresponde à perda em peso sofrida pelo produto quando aquecido em condições nas quais a água é removida. $\mathrm{O}$ aquecimento direto da amostra a $105^{\circ} \mathrm{C}$ é o processo mais usual ${ }^{[18]}$. A análise do teor de umidade das farinhas comerciais (Tabela 1) mostrou que todas as quatro amostras apresentavam-se de acordo com o limite estabelecido pela legislação Brasileira ${ }^{[6]}$ para amidos comerciais, que é definido como no máximo $14 \%$ de umidade.

As farinhas de amido de milho, fécula de batata e trigo apresentaram um teor de umidade semelhante, já a farinha de banana verde apresentou um teor de umidade correspondente a cerca de metade do apresentado pelas demais amostras. Este resultado está de acordo com o apresentado por outros estudos de caracterização de farinha de banana verde, nos quais a obtenção de farinhas de banana verde pelos processos de liofilização e secagem em tambor rotativo apresentaram farinhas com teores de umidade de $2,36 \%$ e $5,46 \%$ respectivamente ${ }^{[19,20]}$.

$\mathrm{O}$ espectro de infravermelho do amido nativo apresenta bandas na região de 2900-3000 $\mathrm{cm}^{-1}$ (correspondente estiramento C-H), em $1163,1150,1124$ e $1103 \mathrm{~cm}^{-1}$, que correspondem ao estiramento $\mathrm{C}-\mathrm{O}$ e C-C com alguma contribuição do estiramento C-OH. As bandas em 1077, 1067, 1047, 1022, 994 e 928 cm$^{-1}$ são atribuídas às deformações $\mathrm{C}-\mathrm{OH}$ e $\mathrm{CH} 2$. O grupo C-O-C (éter) presente em um anel de seis átomos (como no monômero de glucose) absorve em $1150-1085 \mathrm{~cm}^{-1}$ e dependendo de deformação axial (simétrica ou assimétrica) essas bandas irão se deslocar ${ }^{[21]}$.

A espectroscopia de absorção na região do infravermelho foi usada para a caracterização das farinhas. A Figura 1 mostra para as quatro amostras as bandas referentes à deformações moleculares existentes nas moléculas de amido a 3400 e $1650 \mathrm{~cm}^{-1}$, atribuídas ao estiramento e a deformação angular de ligações -OH. Além dessas bandas, é possível observar a banda próximo a $2926 \mathrm{~cm}^{-1}$, atribuída à deformação axial de ligações C-H. As bandas na região $1200 \mathrm{a}$ $1000 \mathrm{~cm}^{-1}$ são consideradas bandas características do amido e são atribuídas a vibrações de deformação axial de C-O em alcoóis e a vibrações de deformação axial do sistema O-C-O.

A análise por técnica de difração de raios $\mathrm{X}$ permite distinguir os três tipos de cristalinidade para os grânulos que, dependendo de sua forma e estrutura cristalina, denominam-se A, B e $\mathrm{C}^{[22,23]}$. Estes padrões de cristalinidade dependem, em parte, do comprimento das cadeias de amilopectina, da densidade de empacotamento dentro dos grânulos, bem como da presença de água ${ }^{[24]}$. Amidos com cristalinidade do tipo A apresentam picos de intensidade nos ângulos de difração $2 \theta$ em aproximadamente $15,3^{\circ} ; 17,1^{\circ} ; 18,2^{\circ} \mathrm{e}$ $23,5^{\circ}$; tipo B em aproximadamente $5,6^{\circ}, 14,4^{\circ} ; 17,2^{\circ} ; 22,2^{\circ}$ e $24^{\circ}$; tipo $\mathrm{C}$ em aproximadamente $5,6^{\circ}, 15,3^{\circ} ; 17,3^{\circ}$ e $23,5^{\circ}[24]$. Há ainda, um quarto tipo de cristalinidade, o tipo $\mathrm{V}$, formado pela cristalização da amilose com lipídios, que apresenta picos de intensidade nos ângulos de difração $2 \theta$ em aproximadamente $12,6^{\circ}, 13,2^{\circ}$,

Tabela 1. Porcentual de umidade encontrado para as farinhas comerciais.

\begin{tabular}{lc}
\hline Farinha & Teor de umidade $(\mathbf{g} / \mathbf{1 0 0} \mathbf{g})$ \\
\hline Amido de milho & $7,03 \pm 0,14$ \\
Fécula de batata & $7,37 \pm 0,16$ \\
Banana verde & $3,14 \pm 0,29$ \\
Trigo & $7,67 \pm 0,22$ \\
\hline
\end{tabular}

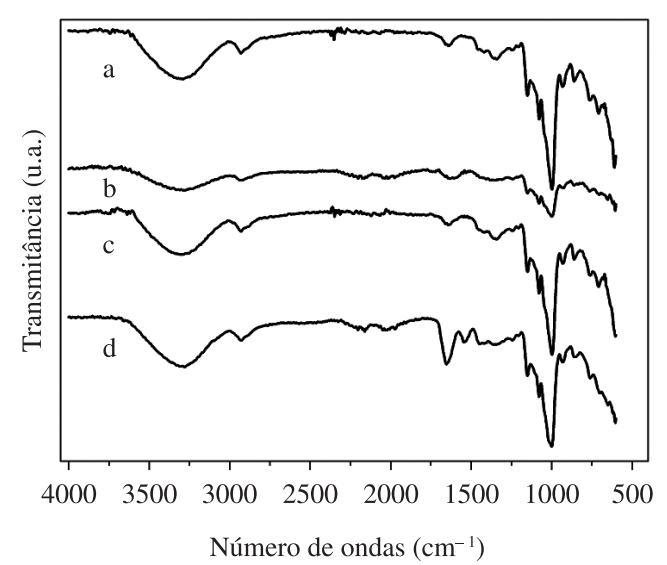

Figura 1. Espectros de absorção na região do infravermelho das farinhas comerciais de (a) amido de milho, (b) fécula de batata, (c) banana verde e (d) trigo. 
$19,4^{\circ}$ e $20,6^{\circ[25]}$. As farinhas de amido de milho e trigo (Figura 2) apresentaram difratogramas de raios $\mathrm{x}$ com picos em $2 \theta$ referentes ao padrão de cristalinidade do tipo $\mathrm{A}$, já as farinhas de fécula de batata e de farinha de banana apresentaram picos característicos de cristalinidade do tipo B. O milho e o trigo são cereais e a batata é denominada tubérculo, portanto, os resultados encontrados estão de acordo com os dados relatados por Van Soest ${ }^{[3]}$, no qual o autor afirma que os amidos de cereais apresentam cristalinidade do tipo A, os amidos de tubérculos cristalinidade do tipo B e os amidos de raízes e sementes cristalinidade do tipo C. Embora não haja um padrão de cristalinidade atribuído aos amidos oriundos de frutos, o resultado obtido para a farinha de banana, como cristalinidade do tipo B é confirmado por estudos anteriores ${ }^{[26,27]}$.

A Tabela 2 mostra os graus de cristalinidade calculados para as farinhas comerciais. O grau de cristalinidade dos amidos é proporcional à sua composição, os amidos regulares são compostos de $70 \%$ a $80 \%$ por amilopectina e de 20 a $30 \%$ por amilose ${ }^{[28,29]}$. Os graus de cristalinidade encontrados estão de acordo com a literatura, que diz que o grau de cristalinidade $(\mathrm{Xc})$ para os amidos regulares deve estar compreendido entre $20 \%$ e $45 \%{ }^{[3]}$.

A partir das curvas obtidas por análise termogravimétrica das farinhas comerciais (Figura 3) foi possível determinar a temperatura inicial média de degradação e o porcentual de materiais inorgânicos nas amostras.

A farinha de amido de milho apresentou a temperatura inicial média de degradação em aproximadamente $292{ }^{\circ} \mathrm{C}$, a fécula de batata em $286{ }^{\circ} \mathrm{C}$, a farinha de banana verde em $256^{\circ} \mathrm{C}$ e a farinha de trigo em $282^{\circ} \mathrm{C}$.

A partir da obtenção da curva derivada do termograma (DTG) foi possível determinar as temperaturas nas quais a velocidade degradação é máxima para cada uma das amostras.

A farinha de amido de milho apresentou duas etapas de perda de massa o primeiro referente à saída de água da amostra e a segunda relativa à degradação do polissacarídeo, porém a DTG mostrou, além do evento de desidratação em aproximadamente $44{ }^{\circ} \mathrm{C}$, dois eventos de degradação em aproximadamente $278{ }^{\circ} \mathrm{C}$ e $308^{\circ} \mathrm{C}$. O mesmo perfil foi observado para a fécula de batata, cujos eventos ocorreram em aproximadamente $49^{\circ} \mathrm{C}, 277^{\circ} \mathrm{C}$ e $302^{\circ} \mathrm{C}$.

Tabela 2. Grau de cristalinidade calculado para as farinhas comerciais.

\begin{tabular}{lc}
\hline \multicolumn{1}{c}{ Farinha } & Grau de cristalinidade (\%) Xc \\
\hline Amido de milho & 32 \\
Fécula de batata & 36 \\
Banana verde & 33 \\
Trigo & 26 \\
\hline
\end{tabular}

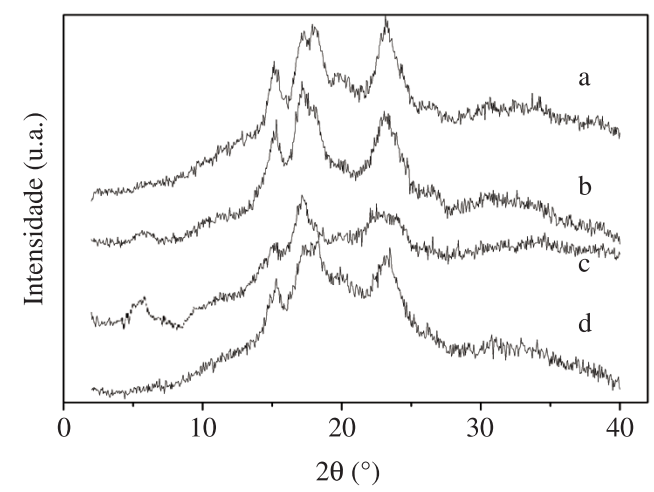

Figura 2. Difratogramas de raios $X$ das farinhas comerciais de (a) amido de milho, (b) fécula de batata, (c) banana verde e (d) trigo.
O termograma obtido para a farinha de banana verde apresentou três etapas de perda de massa, o evento de desidratação ocorreu em aproximadamente $45^{\circ} \mathrm{C}$ e os eventos de degradação ocorreram em aproximadamente $282^{\circ} \mathrm{C}$ e $467{ }^{\circ} \mathrm{C}$.

$\mathrm{O}$ termograma obtido para a farinha de trigo apresentou apenas duas etapas de perda de massa e o evento de desidratação ocorreu em aproximadamente $44^{\circ}$ e o evento de degradação ocorreu em aproximadamente $304^{\circ} \mathrm{C}$.

O primeiro evento observado nas DTGs (entre $44^{\circ} \mathrm{Ce} 49^{\circ} \mathrm{C}$ ) está relacionado à saída de água das amostras, uma vez que as cadeias de glicose que constituem o amido são interligadas e enroladas sobre si mesmas e apresentam diferentes graus de hidratação. As reações hidrotérmicas abordam as relações dos grânulos de amido com a água e as variações de temperatura a partir de $30{ }^{\circ} \mathrm{C}$ provocam alterações estruturais ${ }^{[30]}$.
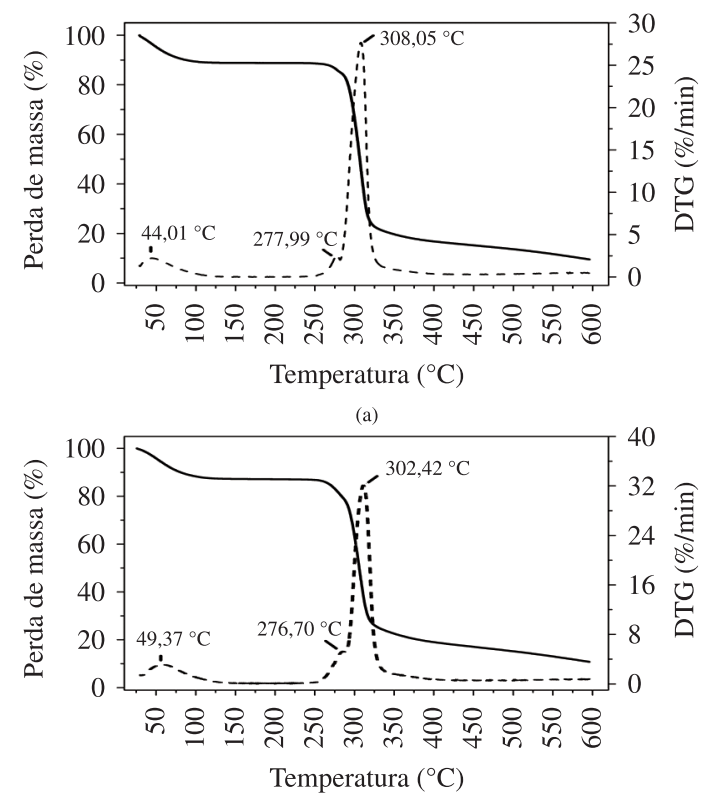

(b)

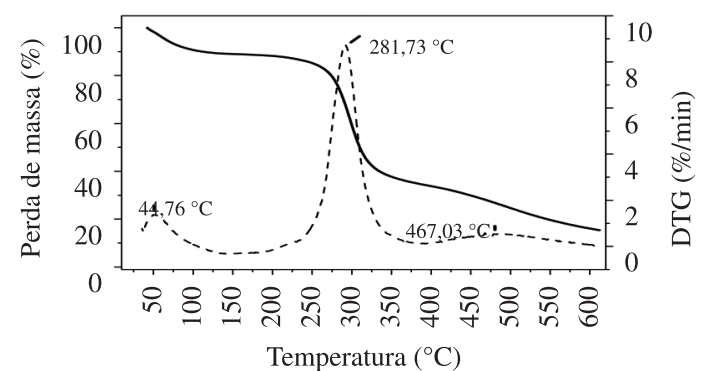

(c)

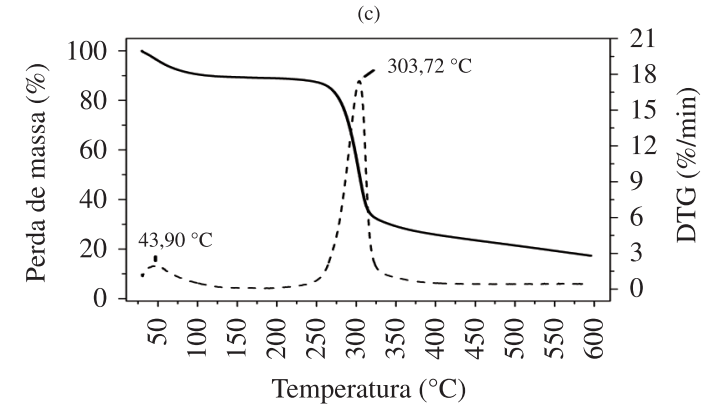

(d)

Figura 3. Termogramas das farinhas comerciais de amido de milho (a), fécula de batata (b), banana verde (c) e trigo (d). 
O evento de degradação do polissacarídeo ocorre por despolimerização quando a temperatura aplicada excede os $300^{\circ} \mathrm{C}^{[31-34]}$.

A partir da análise termogravimétrica é possível determinar o teor de inorgânicos nas amostras ${ }^{[35]}$. Contudo, a análise realizada sob atmosfera controlada de $\mathrm{N}_{2}$ não forneceu dados compatíveis com os valores de teor de cinzas obtidos por determinação convencional ${ }^{[18]}$, como mostra a Tabela 3 .

Por meio das medidas de relaxação longitudinal é possível observar os domínios de núcleos de hidrogênio das amostras ${ }^{[9]}$. A Tabela 4 mostra os tempos de relaxação para as amostras de farinhas comerciais. As quatro amostras apresentaram duas regiões de mobilidade distintas, ou seja, com diferentes graus de mobilidade.

A farinha de amido de milho apresentou uma região de alta mobilidade $(6 \mathrm{~ms})$ relacionada à relaxação dos núcleos de hidrogênio da água presentes na amostra e uma segunda região com menor mobilidade $(93 \mathrm{~ms})$ referente à relaxação dos núcleos de hidrogênio do polissacarídeo e possíveis proteínas presentes na amostra. A farinha de batata apresentou uma região de mobilidade elevada (5 ms) referente aos núcleos hidrogênios da água presente na amostra e outra de mobilidade mais restrita $(57 \mathrm{~ms})$, relacionado à presença de ácidos graxos e lipídios nesta amostra, e um domínio referente ao polissacarídeo ( $88 \mathrm{~ms}$ ), tal como observado para o amido de milho.

A farinha de banana verde a farinha de banana verde apresentou uma região com maior mobilidade, referente à presença de água na amostra ( $5 \mathrm{~ms})$ e uma menos móvel $(48 \mathrm{~ms})$, provavelmente referente ao domínio de polissacarídeo. Este menor tempo de

Tabela 3. Porcentual de inorgânicos encontrado por análise de TGA e porcentual de cinzas encontrado para as farinhas comerciais.

\begin{tabular}{lcc}
\hline Farinha & $\begin{array}{c}\text { Teor de inorgânicos } \\
(\mathbf{g} / \mathbf{1 0 0} \mathbf{g})\end{array}$ & $\begin{array}{c}\text { Teor de cinzas } \\
(\mathbf{g} / \mathbf{1 0 0} \mathbf{~ g})\end{array}$ \\
\hline Amido de milho & 9,27 & $0,96 \pm 0,09$ \\
Fécula de batata & 10,81 & $0,87 \pm 0,23$ \\
Banana verde & 15,34 & $2,03 \pm 1,02$ \\
Trigo & 17,31 & $0,72 \pm 0,17$ \\
\hline
\end{tabular}

Tabela 4. Tempo de relaxação longitudinal $\left(\mathrm{T}_{1} \mathrm{H}\right)$ e porcentual dos domínios dos núcleos de hidrogênio para as farinhas comerciais.

\begin{tabular}{lcc}
\hline \multicolumn{1}{c}{ Amostra } & $\mathbf{T}_{\mathbf{1}} \mathbf{H}(\mathbf{m s})$ & $\mathbf{\%}$ \\
\hline \multirow{2}{*}{ Amido de milho } & 6 & 3 \\
& 93 & 97 \\
\hline \multirow{3}{*}{ Fécula de batata } & 5 & 5 \\
& 57 & 33 \\
& 88 & 62 \\
\hline \multirow{2}{*}{ Banana verde } & 5 & 14 \\
& 48 & 86 \\
\hline \multirow{2}{*}{ Trigo } & 4 & 6 \\
& 96 & 94 \\
\hline
\end{tabular}

Tabela 5. Tempo de relaxação longitudinal $\left(\mathrm{T}_{1} \mathrm{H}\right)$ e porcentual dos domínios dos núcleos de hidrogênio para as farinhas comerciais após extração de lipídios.

\begin{tabular}{lrr}
\hline \multicolumn{1}{c}{ Amostra } & $\mathbf{T}_{\mathbf{1}} \mathbf{H}(\mathbf{m s})$ & $\boldsymbol{\%}$ \\
\hline \multirow{2}{*}{ Amido de milho } & 3,2 & 1,95 \\
& 93,6 & 98,05 \\
\hline \multirow{3}{*}{ Fécula de batata } & 4,9 & 4,75 \\
& 66,8 & 48,82 \\
& 90,9 & 46,43 \\
\hline \multirow{2}{*}{ Banana verde } & 5,6 & 11,82 \\
& 54,1 & 88,18 \\
\hline \multirow{2}{*}{ Trigo } & 3,1 & 2,69 \\
& 98,4 & 97,31 \\
\hline
\end{tabular}

relaxação caracteriza um domínio constituído por polissacarídeos com maior mobilidade quando comparado às demais farinhas, provavelmente porque não é um amido purificado e apresenta todos os outros componentes presentes na banana.

A farinha de trigo apresentou uma região de mobilidade referente aos núcleos de hidrogênio das moléculas de água (4 ms) e uma outra região mais rígida $(96 \mathrm{~ms})$ referente aos polissacarídeos.

Com a finalidade de observar se os tempos de relaxação destas amostras estão relacionados com o teor de óleo contido nas farinhas, as amostras foram submetidas à extração lipídica em extrator de soxhlet, por 24 horas na qual o solvente utilizado foi o n-hexano ${ }^{[18]}$. A Tabela 5 mostra os tempos de relaxação das amostras de farinha após a extração de óleo.

Não houve mudança significativa nos tempos de relaxação quando comparadas as amostras integrais às amostras que sofreram extração, portanto os tempos de relaxação dos domínios encontrados nas farinhas não sofrem influência do teor de óleo nas amostras. Neste caso, as regiões de mobilidade podem estar relacionadas à composição dos amidos presentes nas farinhas, ou seja, à proporção de amilose/amilopectina encontrada nestes polissacarídeos ${ }^{[25]}$. Porém, é necessária a realização de novos estudos para maiores esclarecimentos.

\section{Conclusões}

As farinhas comerciais com base em amido estavam de acordo com o preconizado pela legislação brasileira em relação ao teor de umidade e apresentaram grau de cristalinidade compatível com amostras de amido regulares (não modificados).

A análise de espectroscopia de absorção na região do infravermelho apresentou bandas características para amido nas amostras de farinha comerciais.

A técnica de caracterização por difração de raios-X permitiu observar os diferentes tipos e graus de cristalinidade para os amidos das amostras estudadas.

A caracterização por análise termogravimétrica permitiu a determinação da temperatura inicial média de degradação e a obtenção da DTG forneceu os valores nos quais ocorrem o fenômeno de desidratação e os eventos de degradação dos polissacarídeos.

As medidas de tempo de relaxação longitudinal (T1H) obtidas por ressonância magnética nuclear de baixo campo forneceram informações quanto à dinâmica molecular dos componentes das farinhas.

Estudos complementares a cerca da dinâmica molecular e da composição dos amidos de farinhas comerciais, sobretudo a relação amilose/amilopectina, se fazem necessários para uma melhor compreensão do comportamento destes polissacarídeos.

\section{Referências Bibliográficas}

1. Doane, W. M.; Swanson, C. \& Fanta, G. - "Emerging polymeric materials based on starch", in: "Emerging technologies for materials and chemicals from biomass", p.469, R. M. Rowell, T. P. Schietz, R. Narayan, (ed.), American Chemical Society, Washington (1992). http:// dx.doi.org/10.1021/bk-1992-0476.ch013

2. Poutanen, K. \& Forssel, P. - Trends Polymer Sci, 4, p.128 (1996).

3. Van Soest, J. J. G \& Vliegenthart, J. F. G. - Tibtech, 15, p.208 (1997). http://dx.doi.org/10.1016/S0167-7799(97)01021-4

4. Whilstler, R. L. \& Daniel, J. R. - "Encyclopedia of chemical technology", John-Wiley, New York (1982).

5. Caballero, B. - "Guide to Nutritional Supplements", Academic Press, New York (1982).

6. Agência Nacional de Vigilância Sanitária. Gerência-Geral Alimentos, Resolução - “CNNPA n 12, de 1978”, Diário Oficial da República 
Federativa do Brasil, Brasília, 24 jul. 1978. Disponível em: <http:// www.anvisa.gov.br/legis/resol/12_78.pdf>

7. Nascimento, A. M. R. - "Estudo do látex e fruto da sorva pela espectroscopia de ressonância magnética nuclear", Tese de Doutorado, Universidade Federal do Rio de Janeiro, Brasil (2006).

8. Silva, N. M. \& Tavares, M. I. B. - Polímeros, 4, p.43 (1994).

9. Preto, M.; Tavares, M. I. B. \& Silva, P. E. - Polym. Test., 26, p.501 (2007). http://dx.doi.org/10.1016/j.polymertesting.2007.01.009

10. Tavares, M. I. B. - Polym. Test., 19, p.2000 (2000).

11. Tavares, M. I. B. \& Mendes, L. C. - Polym. Test., 19, p.399 (2000). http://dx.doi.org/10.1016/S0142-9418(99)00010-0

12. Resende, D. K.; Dornelas, C. B.; Tavares, M. I. B.; Gomes, A. S.; Moreira, L. A.; Cabral, L. M.\& Simeoni, L. A. - Polímeros, 20, p.231 (2010). http://dx.doi.org/10.1590/S0104-14282010005000031

13. Bertram, H. C.; Karlsson, A. H.; Rasmussen, M.; Pedersen, O. D.; Donstrup, S. D. \& Andersen, H. J. - J. Agr. Food Chem., 49, p.3092 (2001). PMid:11410014. http://dx.doi.org/10.1021/jf001402t

14. Tang, H. R.; Godward, J. \& Hills, B. - Carbohyd. Polym., 43, p.375 (2000). http://dx.doi.org/10.1016/S0144-8617(00)00183-1

15. Thybo, A. K; Bechman, I. E.; Martens, M. \& Engelsen, S. B. - Lebensm. Wiss. Technol., 23, p.103 (2000). http://dx.doi.org/10.1006/ fstl.1999.0623

16. Maciel, P. M. C. - "Preparação da mistura cumbaru-PVP-colágeno e caracterização por espectroscopia de ressonância magnética nuclear no estado sólido", Tese de Doutorado, Universidade Federal do Rio de Janeiro, Brasil (2008).

17. Maciel, P. D. C. \& Tavares, M. I. B - J. Appl. Polym. Sci., 50, p.116 (2010).

18. Zenebon, O.; Pascuet, N. S. \& Tiglea, P. - "Métodos físico-químicos para análise de alimentos", Instituto Adolfo Lutz, São Paulo (2008).

19. Borges, A. M.; Pereira, J. \& Lucena, E. M. P. - Ciênc. Tecnol. Aliment., 29, p.333 (2009). http://dx.doi.org/10.1590/S0101-20612009000200015

20. Pacheco-Delahaye, E. \& Testa, G. - INCI., 30, p.300 (2005).

21. Silverstein, R. M.; Bassler, G. C. \& Morrill, T. C. - "Infrared Spectrometry". in: Spectrometric Identification of Organic Compound, p.91, John Wiley \& Sons, Singapura (1991).
22. Cereda, M. P - "Propriedades gerais do amido", Fundação Cargill, São Paulo (2001).

23. Zobel, H. F. - "X-ray analysis of starch granules". in: Methods in carbohydrates chemistry, p.109-143, Academic Press, New York (1964).

24. Sajilata, M. G.; Singhal, R. S. \& Kulkarni, P. R. - Compr Rev. Food Sci. Food Safety., 5, p.1, (2006). http://dx.doi. org/10.1111/j.1541-4337.2006.tb00076.x

25. Corradini, E.; Lotti, C.; Medeiros, E. S.; Carvalho, A. J. F.; Curvelo, A. A. S. \& Mattoso, L. H. C. - Polímeros, 15, p.268 (2005).

26. Lobo, A. R. \& Silva, G. M. - Rev. Nutr., 16, p.219 (2003).

27. Elfstrand, L.; Frigard, T.; Andersson, R.; Eliasson, A. C.; Jonsson, M.; Reslow, M. \& Wahlgren, M. - Carb. Polym., 57, p.389 (2004). http:// dx.doi.org/10.1016/j.carbpol.2004.05.018

28. Lima, F. F. \& Andrade, C. T. - Ultrason. Sonochem., 17, p.637 (2010). Pmid:20133177. http://dx.doi.org/10.1016/j.ultsonch.2010.01.001

29. Morisson, W. R. \& Laignelet, B. - J. Cereal Sci., 1, p.9 (1983). http:// dx.doi.org/10.1016/S0733-5210(83)80004-6

30. Cereda, M. \& Vilpoux, O.F. - "Tecnologia, usos e potencialidades de tuberosas amiláceas latino americanas”, Fundação Cargill, São Paulo (2003).

31. Silva, S. S.; Carvalho, R. R.; Fonseca, J. L. C. \& Garcia, R. B. - Polímeros, Abr/Jun, p.25 (1998). http://dx.doi.org/10.1590/S010414281998000200005

32. Aggarwall, P. \& Dollimore, D. - Therm. Acta, 319, p.17, (1998). http:// dx.doi.org/10.1016/S0040-6031(98)00355-4

33. Rudnik, E.; Matuschek, G.; Milanov, N.; Kettrup, A. - J. Therm. Anal. Calorim., 85, p.267 (2006). http://dx.doi.org/10.1007/s10973-005-7274-7

34. Guinesi L. S., Róz, A. L.; Corradini, E., Mattoso L. H. C., Teixeira E. M. \& Curvelo A. A. S. - Therm. Acta, 447, p.190 (2006). http://dx.doi. org/10.1016/j.tca.2006.06.002

35. Araújo, A. A. S.; Mercuri, L. P.; Seixas, S. R. S. Storpirtis, S.; Matos, J. R. - Braz. J. Pharm. Sci., 42, p.269 (2006).

Enviado: 27/06/11

Reenviado: $13 / 11 / 11$

Aceito: 08/02/12 